\title{
Toy Models of a Nonassociative Quantum Mechanics
}

\author{
Vladimir Dzhunushaliev \\ Department of Physics and Microelectronics Engineering, Kyrgyz-Russian Slavic University, \\ 44 Kievskaya Street, Bishkek 720021, Kyrgyzstan \\ Correspondence should be addressed to Vladimir Dzhunushaliev, dzhun@krsu.edu.kg
}

Received 19 July 2007; Revised 8 September 2007; Accepted 18 September 2007

Recommended by George Siopsis

Toy models of a nonassociative quantum mechanics are presented. The Heisenberg equation of motion is modified using a nonassociative commutator. Possible physical applications of a nonassociative quantum mechanics are considered. The idea is discussed that a nonassociative algebra could be the operator language for the nonperturbative quantum theory. In such approach the nonperturbative quantum theory has observables and unobservables quantities.

Copyright ( 12007 Vladimir Dzhunushaliev. This is an open access article distributed under the Creative Commons Attribution License, which permits unrestricted use, distribution, and reproduction in any medium, provided the original work is properly cited.

\section{Introduction}

In [1], the attempt was made to obtain a possible generalization of quantum mechanics on any numbers including nonassociative numbers: octonions. In [2], the author applies nonassociative algebras to physics. This book covers topics ranging from algebras of observables in quantum mechanics, to angular momentum and octonions, division algebras, triple-linear products, and Yang-Baxter equations. The nonassociative gauge theoretic reformulation of Einstein's general relativity theory is also discussed. In [3], one can find the review of mathematical definitions and physical applications for the octonions. The modern applications of the nonassociativity in physics are as follows: in $[4,5]$ it is shown that the requirement that finite translations be associative leads to Dirac's monopole quantization condition; in [6, 7] Dirac's operator and Maxwell's equations are derived in the algebra of split-octonions.

In this paper, we attempt to give toy models of a nonassociative quantum mechanics using finite dimensional nonassociative algebras-octonions or sedenions. In the previous paper [8], we have shown that in a nonassociative quantum theory the observables can be presented only by elements of an associative subalgebra of a nonassociative algebra of nonperturbative 
quantum operators. Unfortunately, we cannot now present any model of nonassociative quantum theory since building of such nonassociative infinite dimensional algebra is a very complicated mathematical problem. But in this paper we present a toy model of nonassociative quantum mechanics. We can do that using an analogy with the standard quantum mechanics with the spin: if the relevant degrees of freedom for us are spin degrees of freedom only (the coordinate dependence of a wave function is not important), then we will have a qubit quantum system. The qubit quantum mechanics is much simpler than the standard quantum mechanics with Pauli equation.

In this paper, we will show that there exists a finite dimensional nonassociative algebra (octonions or sedenions) that has an associative subalgebra (quaternions, biquaternions). The associative subalgebra may have a noncommutative subalgebra (quaternions) and a commutative subalgebra. The quantum states of the noncommutative subalgebra are qubits. Three eigenvectors of the commutative subalgebra one can identify with three qubit fermion generations.

Why can a nonassociative quantum theory be interesting? The reason is that it could be a candidate for a nonperturbative quantum theory formulated on the operator language. Generally speaking (according to [8]) the elements of such algebra are unobservables but if in such nonassociative algebra exists an associative subalgebra, then their elements are observables.

Thus the goal of this paper is to show that one can find a nonassociative finite dimensional algebra having an associative subalgebra (which elements are observables only) and to show that one can correctly define the Heisenberg equation of motion using the nonassociativity property.

\section{Nonassociative quantum dynamics}

In this section, we would like to present a toy model of quantum mechanics realized on a finite dimensional associative subalgebra (quaternions $\mathbb{Q}$ or biquaternions $\mathbb{B}$ ) of a nonassociative algebra (octonions $\mathbb{O}$ or sedenions $\mathbb{S}$, resp.).

In the usual associative quantum mechanics, we obtain the time evolution of any operator $\widehat{x}$ from the Heisenberg equation of motion for the Hamiltonian $\widehat{H}$

$$
\frac{d \widehat{x}}{d t}=\mathrm{I}[\widehat{H}, \widehat{x}],
$$

where $[\widehat{x}, \widehat{y}]=\hat{x} \hat{y}-\widehat{y} \hat{x}$ is the commutator and $\mathrm{I}^{2}=-1$; later we will omit ${ }^{\text {. Among many pro- }}$ posed methods for generalizing or modifying the present framework of quantum mechanics, Nambu suggested [9] to modify the Heisenberg equation of motion (2.1) into a triple product equation

$$
\frac{d x}{d t}=\left\{h_{1}, h_{2}, x\right\}
$$

where $\{x, y, z\}$ is a triple-linear product, and we use two Hamiltonian operators $h_{1,2}$, instead of the customary one Hamiltonian as given in (2.1).

Let us define a triple product following [2]. The three-linear product in a vector space $V$ can be identified by a linear mapping

$$
f: V \otimes V \otimes V \longrightarrow V .
$$


For any $x, y, z \in V$, we assign an element $w \in V$, which is linear in each of $x, y, z$, and we write $w=f(x, y, z)=\{x, y, z\}$. The consistency condition

$$
\frac{d}{d t}(x y)=x \frac{d y}{d t}+\frac{d x}{d t} y
$$

for (2.1) leads to

$$
\left\{h_{1}, h_{2}, x y\right\}=x\left\{h_{1}, h_{2}, y\right\}+\left\{h_{1}, h_{2}, x\right\} y \text {, }
$$

where $h_{1,2}, x, y \in V$ and can be any elements of the algebra $V$ (now the vector space $V$ simultaneously is an algebra). In [8], it is shown that if we would like to introduce physical observables in a nonassociative quantum theory, then they can be elements of an associative subalgebra only. Therefore, in contrast with the definition (2.2) of quantum dynamic on a nonassociative algebra $V$ we will propose that the observables $x \in V_{1} \subset V\left(V_{1}\right.$ is an associative subalgebra of a nonassociative algebra $V$ ) and $h_{1,2} \in V_{1} \backslash V$ are nonassociative elements of the algebra $V$.

\subsection{A nonassociative quantum mechanics on quaternions}

Bearing in mind that the quaternions algebra is equivalent to a qubit algebra, one can apply the results of this section to the dynamics of spin, polarized photon, and so on.

Let us introduce a quantum nonassociative dynamics on $\mathbb{Q}$ using full nonassociative algebra $\mathbb{O}$ octonions: $\mathbb{Q} \subset \mathbb{O}$ (in Appendix 5 the definitions for all algebras and multiplication table are given). For this we introduce a nonassociative commutator (n/a-commutator) in the following way:

$$
\left[i_{4}, i_{m+4}, b\right] \equiv i_{4}\left(i_{m+4} b\right)-\left(b i_{4}\right) i_{m+4}, \quad m=1,2,3
$$

generalizing the usual associative commutator $[a b, c]=(a b) c-c(a b)=a b c-c a b$ in the following nonassociative way:

$$
[a, b, c] \equiv a(b c)-(c a) b, \quad a, b \in \mathbb{O}, c \in \mathbb{Q} \backslash \mathbb{O} .
$$

One can check that

$$
\left[i_{4}, i_{m+4}, i_{n}\right]=-2 \varepsilon_{m n k} i_{k}, \quad m, n, k=1,2,3
$$

The nontriviality of $\mathrm{n} / \mathrm{a}$-commutator $\left[i_{4}, i_{m+4}, b\right]$ is that

$$
i_{4}\left(i_{m+4} b\right) \neq\left(i_{4} i_{m+4}\right) b, \quad\left(b i_{4}\right) i_{m+4} \neq b\left(i_{4} i_{m+4}\right) .
$$

The consistency condition (2.4) for (2.1) leads to

$$
[H, x y]=x[H, y]+[H, x] y
$$

which can be easily proved for the associative algebra. But in our case the consistency condition (2.4) leads to

$$
\left[i_{4}, i_{m+4}, b c\right]=b\left[i_{4}, i_{m+4}, c\right]+\left[i_{4}, i_{m+4}, b\right] c, \quad b, c=i_{k} \in \mathbb{Q}, m, k=1,2,3
$$


Table 1: The sedenions multiplication table.

\begin{tabular}{|c|c|c|c|c|c|c|c|c|c|c|c|c|c|c|c|c|}
\hline & 1 & $i_{1}$ & $i_{2}$ & $i_{3}$ & $i_{4}$ & $i_{5}$ & $i_{6}$ & $i_{7}$ & $i_{0}$ & $\epsilon_{1}$ & $\epsilon_{2}$ & $\epsilon_{3}$ & $\epsilon_{4}$ & $\epsilon_{5}$ & $\epsilon_{6}$ & $\epsilon_{7}$ \\
\hline 1 & 1 & $i_{1}$ & $i_{2}$ & $i_{3}$ & $i_{4}$ & $i_{5}$ & $i_{6}$ & $i_{7}$ & $i_{0}$ & $\epsilon_{1}$ & $\epsilon_{2}$ & $\epsilon_{3}$ & $\epsilon_{4}$ & $\epsilon_{5}$ & $\epsilon_{6}$ & $\epsilon$ \\
\hline$i_{1}$ & $\imath_{1}$ & -1 & $i_{3}$ & $-i_{2}$ & $i_{5}$ & $-i_{4}$ & $-i_{7}$ & $i_{6}$ & $-\epsilon_{1}$ & $i_{0}$ & $\epsilon_{3}$ & $-\epsilon_{2}$ & $\epsilon_{5}$ & $-\epsilon_{4}$ & $-\epsilon$ & $\epsilon_{t}$ \\
\hline$i_{2}$ & $i_{2}$ & $-i_{3}$ & -1 & $i_{1}$ & $i_{6}$ & $i_{7}$ & $-i_{4}$ & $-i_{5}$ & $-\epsilon_{2}$ & $-\epsilon_{3}$ & $i_{0}$ & $\epsilon_{1}$ & $\epsilon_{6}$ & $\epsilon_{7}$ & $-\epsilon_{4}$ & - \\
\hline$i_{3}$ & $i_{3}$ & $i_{2}$ & $-i_{1}$ & -1 & $i_{7}$ & $-i_{6}$ & $i_{5}$ & $-i_{4}$ & $-\epsilon_{3}$ & $\epsilon_{2}$ & $-\epsilon_{1}$ & $i_{0}$ & $\epsilon_{7}$ & $-\epsilon_{6}$ & $\epsilon_{5}$ & - \\
\hline$i_{4}$ & $i_{4}$ & $-i_{5}$ & $-i_{6}$ & $-i_{7}$ & -1 & $i_{1}$ & $i_{2}$ & $i_{3}$ & $-\epsilon_{4}$ & $-\epsilon_{5}$ & $-\epsilon_{6}$ & $-\epsilon$ & $i_{0}$ & $\epsilon_{1}$ & $\epsilon_{2}$ & $\epsilon$ \\
\hline$i_{5}$ & $i_{5}$ & $i_{4}$ & $-i_{7}$ & $i_{6}$ & $-i_{1}$ & -1 & $-i_{3}$ & $i_{2}$ & $-\epsilon_{5}$ & $\epsilon_{4}$ & $-\epsilon_{7}$ & $\epsilon_{6}$ & $-\epsilon_{1}$ & $i_{0}$ & $-\epsilon$ & $\epsilon$ \\
\hline$i_{6}$ & $i_{6}$ & $i_{7}$ & $i_{4}$ & $-i_{5}$ & $-i_{2}$ & $i_{3}$ & -1 & $-i_{1}$ & $-\epsilon_{6}$ & $\epsilon_{7}$ & $\epsilon_{4}$ & $-\epsilon_{5}$ & $-\epsilon$ & $\epsilon_{3}$ & $i_{0}$ & $-\epsilon$ \\
\hline$i_{7}$ & $i_{7}$ & $-i_{6}$ & $i_{5}$ & $i_{4}$ & $-i_{3}$ & $-i_{2}$ & $i_{1}$ & -1 & $-\epsilon_{7}$ & $-\epsilon_{6}$ & $\epsilon_{5}$ & $\epsilon_{4}$ & $-\epsilon_{3}$ & $-\epsilon$ & $\epsilon_{1}$ & $i_{0}$ \\
\hline$i_{0}$ & $i_{0}$ & $-\epsilon_{1}$ & $-\epsilon_{2}$ & $-\epsilon_{3}$ & $-\epsilon_{4}$ & $-\epsilon_{5}$ & $-\epsilon_{6}$ & $-\epsilon_{7}$ & -1 & $i_{1}$ & $i_{2}$ & $i_{3}$ & $i_{4}$ & $i_{5}$ & $i_{6}$ & $i_{7}$ \\
\hline$\epsilon_{1}$ & $\epsilon_{1}$ & $i_{0}$ & $\epsilon_{3}$ & $-\epsilon_{2}$ & $\epsilon_{5}$ & $-\epsilon_{4}$ & $-\epsilon_{7}$ & $\epsilon_{6}$ & $i_{1}$ & 1 & $-i_{3}$ & $i_{2}$ & $-i_{5}$ & $i$ & $i_{7}$ & -1 \\
\hline$\epsilon_{2}$ & $\epsilon_{2}$ & $-\epsilon_{3}$ & $i_{0}$ & $\epsilon_{1}$ & $\epsilon_{6}$ & $\epsilon_{7}$ & $-\epsilon_{4}$ & $-\epsilon_{5}$ & $i_{2}$ & $i_{3}$ & 1 & $-i_{1}$ & $-i_{6}$ & $-i_{7}$ & $i_{4}$ & $i_{5}$ \\
\hline$\epsilon_{3}$ & $\epsilon_{3}$ & $\epsilon_{2}$ & $-\epsilon_{1}$ & $i_{0}$ & $\epsilon_{7}$ & $-\epsilon_{6}$ & $\epsilon_{5}$ & $-\epsilon_{4}$ & $i_{3}$ & $-i_{2}$ & $i_{1}$ & 1 & $-i_{7}$ & $i_{6}$ & $-i_{5}$ & $i_{4}$ \\
\hline$\epsilon_{4}$ & $\epsilon_{4}$ & $-\epsilon_{5}$ & $-\epsilon_{6}$ & $-\epsilon_{7}$ & $i_{0}$ & $\epsilon_{1}$ & $\epsilon_{2}$ & $\epsilon_{3}$ & $i_{4}$ & $i_{5}$ & $i_{6}$ & $i_{7}$ & 1 & $-i_{1}$ & $-i_{2}$ & -1 \\
\hline$\epsilon_{5}$ & $\epsilon_{5}$ & $\epsilon_{4}$ & $-\epsilon_{7}$ & $\epsilon_{6}$ & $-\epsilon_{1}$ & $i_{0}$ & $-\epsilon_{3}$ & $\epsilon_{2}$ & $i_{5}$ & $-i_{4}$ & $i_{7}$ & $-i_{6}$ & $i_{1}$ & 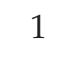 & $i_{3}$ & $-i$ \\
\hline$\epsilon_{6}$ & $\epsilon_{6}$ & $\epsilon_{7}$ & $\epsilon_{4}$ & $-\epsilon_{5}$ & $-\epsilon_{2}$ & $\epsilon_{3}$ & $i_{0}$ & $-\epsilon_{1}$ & $i_{6}$ & $-i_{7}$ & $-i_{4}$ & $i_{5}$ & $i_{2}$ & $-i_{3}$ & 1 & $i_{1}$ \\
\hline$\epsilon_{7}$ & $\epsilon_{7}$ & $-\epsilon_{6}$ & $\epsilon_{5}$ & $\epsilon_{4}$ & $-\epsilon_{3}$ & $-\epsilon_{2}$ & $\epsilon_{1}$ & $i_{0}$ & $i_{7}$ & $i_{6}$ & $-i_{5}$ & $-i_{4}$ & 5 & $i_{2}$ & $-i_{1}$ & 1 \\
\hline
\end{tabular}

and has to be proved. Direct calculations using Table 1 (Appendix 5) show that it is correct that

$$
\left[i_{4}, i_{m+4}, i_{k} i_{l}\right]=-\left[i_{4}, i_{m+4}, i_{l} i_{k}\right]=i_{k}\left[i_{4}, i_{m+4}, i_{l}\right]+\left[i_{4}, i_{m+4}, i_{k}\right] i_{l}, \quad m, k, l=1,2,3 .
$$

The octonion $i_{4}$ can be replaced by any other octonion $n=5,6,7$. It is necessary to note that the consistency condition (2.11) will be destroyed if the numbers $b, c=i_{4,5,6,7} \in \mathbb{O} \backslash \mathbb{Q}$.

For the physical application, let us introduce the following quantities:

$$
\begin{aligned}
h_{m+4} & =i_{m+4} \sqrt{\frac{\mathrm{I} \tilde{\hbar}}{2}}, \quad m=0,1,2,3, \\
\widehat{S}_{k} & =i_{k} \frac{\mathrm{I} \tilde{\hbar}}{2}, \quad k=1,2,3 .
\end{aligned}
$$

Here we introduce a new constant $\tilde{\hbar}$ as it is not evident that in a nonassociative quantum mechanics the Planck constant will be the same; then the $\mathrm{n} / \mathrm{a}$-commutator will have the form

$$
\left[h_{4}, h_{m+4}, \widehat{S}_{n}\right]=-\mathrm{I} \tilde{\hbar} \varepsilon_{m n k} \widehat{S}_{k}, \quad m, n, k,=1,2,3
$$

which should be compared to the qubit dynamic (3.8). Now we can define a nonassociative quantum dynamic of the quantity $\widehat{\vec{S}}=s_{k} \widehat{S}_{k}$ in an external vector field $\widetilde{B}_{m}$ in the following way:

$$
\frac{d \widehat{\vec{S}}}{d t}=\mathrm{I}\left[h_{4},-(\overrightarrow{\widetilde{B}} \cdot \vec{\mu}), \widehat{\vec{S}}\right],
$$


where $\overrightarrow{\widetilde{B}}=\widetilde{B}_{m} \vec{e}_{m}$ is an analog of a magnetic field, $\vec{\mu}=\mu h_{m+4} \vec{e}_{m}$ is an analog of a magnetic dipole for a nonassociative case. Inserting $\widehat{\vec{S}}=s_{k} \widehat{S}_{k}$ into (2.15) leads to

$$
\dot{s}_{k}=-\varepsilon_{m n k} \omega_{m} s_{n}
$$

that describes the rotation of the qubit and $\omega_{m}=\mu B_{m}$ is the angular velocity.

At the end of this section, we would like to mention that full quantum mechanics on the basis of quaternions can be constructed (for details see [16]).

\subsection{A nonassociative quantum mechanics on biquaternions}

A construction similar to that in Section 2.1 can be done for the biquaternions. In this case,

$$
\left[i_{4}, \epsilon_{m+4}, b\right] \equiv i_{4}\left(\epsilon_{m+4} b\right)-\left(b i_{4}\right) \epsilon_{m+4}, \quad m=1,2,3
$$

One can check that

$$
\begin{aligned}
{\left[i_{4}, \epsilon_{m+4}, i_{n}\right] } & =-2 \varepsilon_{m n k} \epsilon_{k}, \\
{\left[i_{4}, \epsilon_{m+4}, \epsilon_{n}\right] } & =2 \varepsilon_{m n k} i_{k}, \quad m, n, k,=1,2,3 .
\end{aligned}
$$

The nontriviality of $\mathrm{n} / \mathrm{a}$-commutator $\left[i_{4}, \epsilon_{m+4}, b\right]$ is that

$$
i_{4}\left(\epsilon_{m+4} b\right) \neq\left(i_{4} \epsilon_{m+4}\right) b, \quad\left(b i_{4}\right) \epsilon_{m+4} \neq b\left(i_{4} \epsilon_{m+4}\right) .
$$

In this case, the consistency condition (2.4) leads to

$$
\left[i_{4}, \epsilon_{m+4}, b c\right]=b\left[i_{4}, \epsilon_{m+4}, c\right]+\left[i_{4}, \epsilon_{m+4}, b\right] c, \quad b, c=i_{k}, \epsilon_{k} \in \mathbb{B}, \quad m, k=1,2,3
$$

and has to be proved. Direct calculations using Table 1 show that it is correct that

$$
\begin{aligned}
{\left[i_{4}, \epsilon_{m+4}, i_{k} i_{l}\right] } & =-\left[i_{4}, \epsilon_{m+4}, i_{l} i_{k}\right]=i_{k}\left[i_{4}, \epsilon_{m+4}, i_{l}\right]+\left[i_{4}, \epsilon_{m+4}, i_{k}\right] i_{l}, \\
{\left[i_{4}, \epsilon_{m+4}, i_{k} \epsilon_{l}\right] } & =-\left[i_{4}, \epsilon_{m+4}, \epsilon_{l} i_{k}\right]=i_{k}\left[i_{4}, \epsilon_{m+4}, \epsilon_{l}\right]+\left[i_{4}, \epsilon_{m+4}, i_{k}\right] \epsilon_{l}, \\
{\left[i_{4}, \epsilon_{m+4}, \epsilon_{k} i_{l}\right] } & =-\left[i_{4}, \epsilon_{m+4}, i_{l} \epsilon_{k}\right]=\epsilon_{k}\left[i_{4}, \epsilon_{m+4}, i_{l}\right]+\left[i_{4}, \epsilon_{m+4}, \epsilon_{k}\right] i_{l}, \\
{\left[i_{4}, \epsilon_{m+4}, \epsilon_{k} \epsilon_{l}\right] } & =-\left[i_{4}, \epsilon_{m+4}, \epsilon_{l} \epsilon_{k}\right]=\epsilon_{k}\left[i_{4}, \epsilon_{m+4}, \epsilon_{l}\right]+\left[i_{4}, \epsilon_{m+4}, \epsilon_{k}\right] \epsilon_{l}, \quad m, k, l=1,2,3
\end{aligned}
$$

The octonion $i_{4}$ can be replaced by any other octonion $i_{n+4}$ with $n=1,2,3$. It is necessary to note that the consistency condition (2.20) will be destroyed if the numbers $b, c=i_{k+4}, \epsilon_{k+4}, k=1,2,3$ belong to $\mathbb{S} \backslash \mathbb{B}$.

For the physical application let us introduce the following quantities:

$$
\begin{aligned}
h_{m+4} & =\epsilon_{m+4} \sqrt{\frac{\mathrm{I} \tilde{\hbar}}{2}}, \quad m=0,1,2,3 ; \\
\widehat{S}_{k} & =i_{k} \frac{\mathrm{I} \tilde{\hbar}}{2}, \quad k=1,2,3 ; \\
\widehat{L}_{k} & =\epsilon_{k} \frac{\mathrm{I} \tilde{\hbar}}{2}, \quad k=1,2,3,
\end{aligned}
$$


then the $\mathrm{n} / \mathrm{a}$-commutator will have the form

$$
\begin{aligned}
& {\left[h_{4}, h_{m+4}, \widehat{S}_{n}\right]=-\mathrm{I} \tilde{\hbar} \varepsilon_{m n k} \widehat{L}_{k}, \quad m, n, k,=1,2,3 ;} \\
& {\left[h_{4}, h_{m+4}, \widehat{L}_{n}\right]=\mathrm{I} \tilde{\hbar} \varepsilon_{m n k} \widehat{S}_{k}, \quad m, n, k,=1,2,3 .}
\end{aligned}
$$

Now we can define a nonassociative quantum dynamic of the quantities $\widehat{\vec{S}}=s_{k} \widehat{S}_{k}, \widehat{\vec{L}}=l_{k} \widehat{L}_{k}$ in an external vector fields $\widetilde{B}_{1,2 ; m}$ in the following way:

$$
\begin{aligned}
& \frac{d \widehat{\vec{S}}}{d t}=\mathrm{I}\left[h_{4},-n_{1}\left(\overrightarrow{\widetilde{B}}_{1} \cdot \vec{\mu}\right), \widehat{\vec{L}}\right], \\
& \frac{d \widehat{\vec{L}}}{d t}=-\mathrm{I}\left[h_{4},-n_{2}\left(\overrightarrow{\widetilde{B}}_{2} \cdot \vec{\mu}\right), \widehat{\vec{S}}\right],
\end{aligned}
$$

where $n_{1,2}= \pm 1$ and describe the sign of the interaction of the fields $\overrightarrow{\widetilde{B}}_{1,2}$ with $\vec{\mu}_{1,2}$ and with the same definitions $\overrightarrow{\widetilde{B}}_{1,2}=\widetilde{B}_{1,2 ; m} \vec{e}_{m}$ and $\vec{\mu}=\mu h_{m+4} \vec{e}_{m}$ as in the previous section. Inserting $\widehat{\vec{S}}=s_{k} \widehat{S}_{k}$ and $\widehat{\vec{L}}=l_{k} \widehat{L}_{k}$ into (2.15) leads to

$$
\begin{aligned}
& \dot{s}_{k}=-\varepsilon_{m n k} \omega_{m} l_{n}, \\
& \dot{l}_{k}=\varepsilon_{m n k} \omega_{m} s_{n},
\end{aligned}
$$

with the same definition of $\omega_{1,2 ; m}=\mu \widetilde{B}_{1,2 ; m}$ as the angular velocity.

\section{Qubits quantum mechanics}

In this section, we would like to present qubit system where the above-mentioned quantum operators from an associative subalgebra of a nonassociative algebra could be operated. Here we follow the textbook [10]. A qubit is a quantum-mechanical two-state system. A canonical example of a qubit is provided by the spin of a spin-1/2 particle, polarized photon, and so on.

Many investigations of quantum systems do not require a "complete" description of the state. For example, one often neglects the position and momentum of a particle when one is only interested in the "inner degrees of freedom" related to the spin. This simplifies the description considerably, because the Hilbert space describing the spin of a particle with spin $1 / 2$ is just the two-dimensional complex vector space $\mathbb{C}_{2}$.

Definition 1. A quantum system with a two-dimensional Hilbert space is called a two-state system or a qubit (quantum bit). The vectors in the Hilbert space of a qubit are often called spinors.

With respect to this basis, vectors are represented by column vectors $\mathbb{C}_{2}$, and linear operators are represented by two-by-two matrices. For example, the basis vectors become

$$
\psi_{+}=\left(\begin{array}{l}
1 \\
0
\end{array}\right), \quad \psi_{-}=\left(\begin{array}{l}
0 \\
1
\end{array}\right) .
$$

A general state of a qubit is an arbitrary superposition of the two basis states,

$$
\psi=c_{+} \psi_{+}+c_{-} \psi_{-}=\left(\begin{array}{l}
c_{+} \\
c_{-}
\end{array}\right), \quad \text { with } c_{ \pm} \in \mathbb{C} .
$$


The norm of $\psi$ and the scalar product with $\phi=d_{+} \psi_{+}+d_{-} \psi_{-}$are given by

$$
\|\psi\|^{2}=\left|c_{+}\right|^{2}+\left|c_{-}\right|^{2}, \quad\langle\psi, \phi\rangle=c_{+}^{*} d_{+}+c_{-}^{*} d_{-} .
$$

Any observable has to be represented by a self-adjoint operator. With respect to a chosen orthonormal basis in the Hilbert space of a qubit, observables are thus represented by Hermitian two-by-two matrices: the three Pauli matrices $\vec{\sigma}=\left(\sigma_{1}, \sigma_{2}, \sigma_{3}\right)$ which are the standard representation of the spin observables $S_{1}, S_{2}$, and $S_{3}$, and are as well the representation of quaternions.

The three Pauli matrices together with the two-dimensional unit matrix $\mathbf{1}_{\mathbf{2}}$ form a basis in the four-dimensional real vector space of all Hermitian two-by-two matrices. With respect to an orthonormal basis in $\mathbb{C}^{2}$, any qubit observable $Q$ is represented by a linear combination of Pauli matrices

$$
Q=\frac{1}{2}\left(a_{0} \mathbf{1}_{2}+\sum_{k=1}^{3} a_{k} \sigma_{k}\right)=\frac{1}{2}\left(\begin{array}{cc}
a_{0}+a_{3} & a_{1}-\mathrm{I} a_{2} \\
a_{1}+\mathrm{I} a_{2} & a_{0}-a_{3}
\end{array}\right)
$$

with real coefficients $a_{0}, \ldots, a_{3}$. It is necessary to note the generation relation for the algebra of Pauli matrices

$$
\left[\sigma_{i}, \sigma_{j}\right]=2 \mathrm{I} \epsilon_{i j k} \sigma_{k}
$$

One can introduce the spin operators $\widehat{S}_{i}=(\hbar / 2) \sigma_{i}$ and then (3.5) has the form

$$
\left[\widehat{S}_{i}, \widehat{S}_{j}\right]=\mathrm{I} \epsilon_{i j k} \widehat{S}_{k}
$$

A general time-independent qubit Hamiltonian has the form

$$
H=a_{0} \mathbf{1}_{2}+\vec{\omega} \vec{\sigma}
$$

where $\vec{\omega}=e \vec{B} / m c$ is the angular velocity speed, $e$ and $m$ is the charge and mass of a particle, and $\vec{B}$ is a magnetic field. The dynamic of the spin $\widehat{\vec{S}}=s_{i} \widehat{S}_{i}$ is described in the following way:

$$
\frac{d \widehat{\vec{S}}}{d t}=\mathrm{I}[H, \widehat{\vec{S}}]
$$

or

$$
\dot{s}_{k}=\varepsilon_{i j k} \omega_{i} s_{j}
$$

that describes the rotation of the qubit.

\section{Possible physical applications}

In Section 2, we presented a nonassociative quantum dynamic. We suppose that the nonassociative quantization procedure could be applied of a nonperturbative quantization for a field theory. The nonassociative quantum dynamic presented in Section 2 can be an approximation in this direction. Now we would like to present a few possible physical applications of such nonassociative quantum dynamic. 


\subsection{Anomalous qubit rotation}

Let us consider (2.16) describing the qubit rotation under action of an external constant field $\overrightarrow{\widetilde{B}}=(0,0, \widetilde{B})$ (which cannot be a magnetic field in our nonperturbative case). Then we have the following equations:

$$
\begin{aligned}
& \dot{s}_{x}=\mu \widetilde{B} s_{y}, \\
& \dot{s}_{y}=-\mu \widetilde{B} s_{x},
\end{aligned}
$$

with the solution

$$
\begin{aligned}
& s_{x}=s_{0 x} \sin (\omega t), \\
& s_{y}=s_{0 y} \cos (\omega t) .
\end{aligned}
$$

The solution describes the rotation of qubit around the external constant field $\overrightarrow{\widetilde{B}}$ in the plane $x y$. Comparing (2.16) and (3.9) we see that the rotation of qubit in the nonassociative quantum mechanics is in the opposite direction in comparison with the rotation of spin around the magnetic field in the standard quantum mechanics.

For the extended version of qubit presented in Section 2.2 we use (2.25) with

$$
\begin{gathered}
\widetilde{B}_{1, z}=\widetilde{B}_{1}, \quad \omega_{1, z}=\omega_{1}, \\
\widetilde{B}_{2, z}=\widetilde{B}_{2}, \quad \omega_{2, z}=\omega_{2}, \\
\dot{s}_{x}=\omega_{1} l_{y}, \\
\dot{l}_{y}=\omega_{2} s_{x},
\end{gathered}
$$

with the following solution:

$$
s_{x}=s_{0 x} e^{ \pm t \sqrt{n_{1} n_{2} \omega_{1} \omega_{2}}}, \quad l_{y}=l_{0 y} e^{ \pm t \sqrt{n_{1} n_{2} \omega_{1} \omega_{2}}} .
$$

It seems that the solution with $n_{1}, n_{2}>0$ is physically senseless as in this case we have exponentially increasing/decreasing operators $\widehat{L}$ and $\widehat{S}$. But in the case $n_{1}, n_{2}<0$ we have the rotation of extended qubits in a plane.

\subsection{Fermion qubit generations}

Let us consider the commutative and associative subalgebra $\mathbb{A}$ spanned on the basis $\left(1, i_{3}, \epsilon_{3}, i_{0}\right)$ which is the commutative subalgebra of the noncommutative and associative algebra of biquaternions $\mathbb{A} \subset \mathbb{B} \subset \mathbb{S}$ (for the definitions of biquaternions and sedenions see Appendix 5). The matrix representation of $\mathbb{A}$ is

$$
\begin{aligned}
& i_{3}=\mathrm{I}\left(\begin{array}{cc}
-\sigma_{3} & 0 \\
0 & 1
\end{array}\right)=\mathrm{I}\left(\begin{array}{ccc}
-1 & 0 & 0 \\
0 & 1 & 0 \\
0 & 0 & 1
\end{array}\right), \\
& \epsilon_{3}=\left(\begin{array}{cc}
\sigma_{3} & 0 \\
0 & 1
\end{array}\right)=\left(\begin{array}{ccc}
1 & 0 & 0 \\
0 & -1 & 0 \\
0 & 0 & 1
\end{array}\right) \\
& i_{0}=\mathrm{I}\left(\begin{array}{cc}
-\mathbf{1}_{2} & 0 \\
0 & 1
\end{array}\right)=\mathrm{I}\left(\begin{array}{ccc}
-1 & 0 & 0 \\
0 & -1 & 0 \\
0 & 0 & 1
\end{array}\right) .
\end{aligned}
$$


The basis vectors are

$$
\xi_{1}=\left(\begin{array}{l}
1 \\
0 \\
0
\end{array}\right), \quad \xi_{2}=\left(\begin{array}{l}
0 \\
1 \\
0
\end{array}\right), \quad \xi_{3}=\left(\begin{array}{l}
0 \\
0 \\
1
\end{array}\right)
$$

which are eigenvectors of matrices (4.5). For such a system, we can apply the Heisenberg equation of motion (2.24).

One can say that the index $k$ (by $\xi_{k}$ ) enumerates "a fermion generation" of extended qubits living in the vector space $E$ of the matrix representation of biquaternions. The vector space $E_{1}$ spanned on the basis vectors (4.6) is a vector subspace $E_{1} \subset E$. According to equation of motion (2.25), the generations of extended qubits can be mixed up. But it is correct only if there exists the interaction term $\left(-\overrightarrow{\widetilde{B}}_{2} \cdot \vec{\mu}\right)$; in the opposite case the qubit generations cannot be mixed up.

In the standard model of particle physics [11-13], there are open questions which have not yet found an answer. Chief among these is the fermion family or generation puzzle as to why the first generation of quarks and leptons (up quark, down quark, electron, and electron neutrino) are replicated in two families or generations of increasing mass (the second generation consisting of charm quark, strange quark, muon, and muon neutrino; the third generation consisting of top quark, bottom quark, tau, and tau neutrino). One can presuppose that a nonassociative infinite dimensional quantum theory may shed light on the generation puzzle of fermions.

\section{Outlook}

Thus we have shown that one can generalize the standard finite dimensional quantum mechanics (e.g., qubit quantum mechanics) to a nonassociative finite dimensional quantum mechanics realized on a finite dimensional associative algebra which is a subalgebra of a nonassociative algebra. We have considered two cases: quaternionic and biquaternionic nonassociative qubit quantum mechanics. In both cases, the nonassociativity is realized in the Heisenberg quantum equation of motion: on RHS of corresponding equations the usual commutator is changed on a nonassociative commutator.

The biquaternion version of nonassociative quantum mechanics has commutative $\left(1, i_{0}, \epsilon_{3}, i_{3}\right)$ and noncommutative $\left(1, i_{1}, i_{2}, i_{3}\right)$ observables. It allows us to suppose that an infinite dimensional nonassociative quantum theory will have an associative subalgebra having physical observables with commutators $\left(\widehat{a} \widehat{a}^{\dagger}-\widehat{a}^{\dagger} \widehat{a}=\mathrm{I} \hbar\right)$ and anticommutators $\left(\widehat{f} \widehat{f}^{\dagger}+\widehat{f}^{\dagger} \hat{f}=\mathrm{I} \hbar\right)$. Probably it means that the unification of bosons and fermions can be done not only on the basis of supersymmetry but in a nonassociative quantum theory as well.

Now we would like to list the results and properties of the nonassociative quantum mechanics discussed here.

(i) Two examples of a finite dimensional quantum mechanics are presented.

(ii) The Heisenberg quantum equation of motion is essentially nonassociative.

(iii) The nonassociativity leads to the fact that the usual Hamiltonian cannot be introduced as the product of two operators.

(iv) Generally speaking the nonassociative factors $\left(i_{4}, i_{m+4}\right)$ or $\left(i_{4}, \epsilon_{m+4}\right)$ are unobservable physical quantities that remind hidden parameters in the theory with hidden parameters. 
(v) The nonassociative quantum theory can be an alternative one to supersymmetric theories.

(vi) In [8], it is shown that the nonassociative quantum theory can describe nonlocal objects like strings and so on.

\section{Appendix}

\section{Sedenions}

Sedenions $[14,15]$ form an algebra with nonassociative but alternative multiplication and a multiplicative modulus. It consists of one real axis (to basis 1), eight imaginary axes (to bases $i_{n}$ with $i_{n}^{2}=-1, n=0, \ldots, 7$ ), and seven real axes (to bases $\epsilon_{n}$ with $\epsilon_{n}^{2}=+1, n=1, \ldots, 7$ ). The multiplication table is given in Table 1 . The sedenions nonassociative algebra contains the following important subalgebras:

(i) the associative quaternion subalgebra $\mathbb{Q}$ with $i_{n}, n=1,2,3$;

(ii) the associative biquaternion subalgebra $\mathbb{B}$ with $i_{0}, i_{n}, \epsilon_{n}, n=1,2,3$;

(iii) the nonassociative octonion subalgebra $\mathbb{O}$ with $i_{n}, n=0, \ldots, 7$.

\section{References}

[1] P. Jordan, J. von Neumann, and E. Wigner, "On an algebraic generalization of the quantum mechanical formalism," Annals of Mathematics, vol. 35, no. 1, pp. 29-64, 1934.

[2] S. Okubo, Introduction to Octonion and Other Non-Associative Algebras in Physics, Montroll Memorial Lecture Series in Mathematical Physics, Cambridge University Press, Cambridge, UK, 1995.

[3] J. C. Baez, "The octonions," Bulletin of the American Mathematical Society, vol. 39, no. 2, pp. 145-205, 2002.

[4] B. Grossman, "A 3-cocycle in quantum mechanics," Physics Letters B, vol. 152, no. 1-2, pp. 93-97, 1985.

[5] R. Jackiw, "Three-cocycle in mathematics and physics," Physical Review Letters, vol. 54, no. 3, pp. 159$162,1985$.

[6] M. Gogberashvili, “Octonionic version of Dirac equations," International Journal of Modern Physics A, vol. 21, no. 17, pp. 3513-3523, 2006.

[7] M. Gogberashvili, “Octonionic electrodynamics," Journal of Physics A, vol. 39, no. 22, pp. 7099-7104, 2006.

[8] V. Dzhunushaliev, "Observables and unobservables in a non-associative quantum theory," preprint, 2007, http:/ /arxiv.org/abs/quant-ph/0702263.

[9] Y. Nambu, "Generalized hamiltonian dynamics," Physical Review D, vol. 7, no. 8, pp. 2405-2412, 1973.

[10] B. Thaller, Advanced Visual Quantum Mechanics, Springer Science \& Business Media, New York, NY, USA, 2005.

[11] S. L. Glashow, "Partial-symmetries of weak interactions," Nuclear Physics, vol. 22, no. 4, pp. 579-588, 1961.

[12] S. Weinberg, "A model of leptons," Physical Review Letters, vol. 19, no. 21, pp. 1264-1266, 1967.

[13] A. Salam, "Relativistic groups and analiticity," in Proceedings of the 8th Nobel Symposium on Elementary Particle Theory, N. Svartholm, Ed., p. 367, Almquist \& Wiksell, Stockholm, Sweden, May 1968.

[14] K. Carmody, "Circular and hyperbolic quaternions, octonions, and sedenions," Applied Mathematics and Computation, vol. 28, no. 1, pp. 47-72, 1988.

[15] K. Carmody, "Circular and hyperbolic quaternions, octonions, and sedenions-further results," Applied Mathematics and Computation, vol. 84, no. 1, pp. 27-47, 1997.

[16] S. L. Adler, Quaternionic Quantum Mechanics and Quantum Fields, Oxford University Press, Oxford, UK, 2001. 

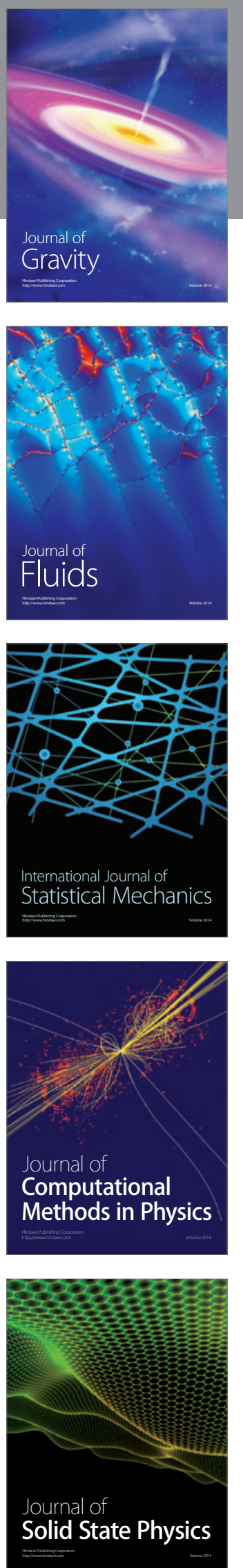

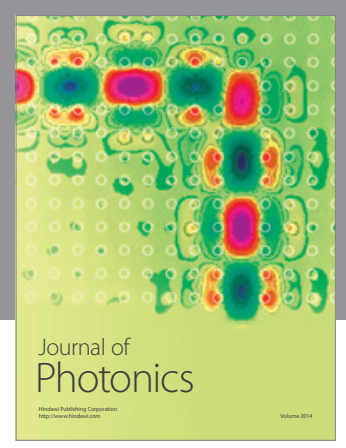

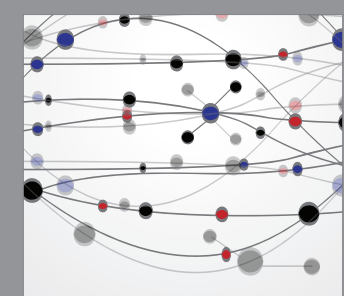

The Scientific World Journal
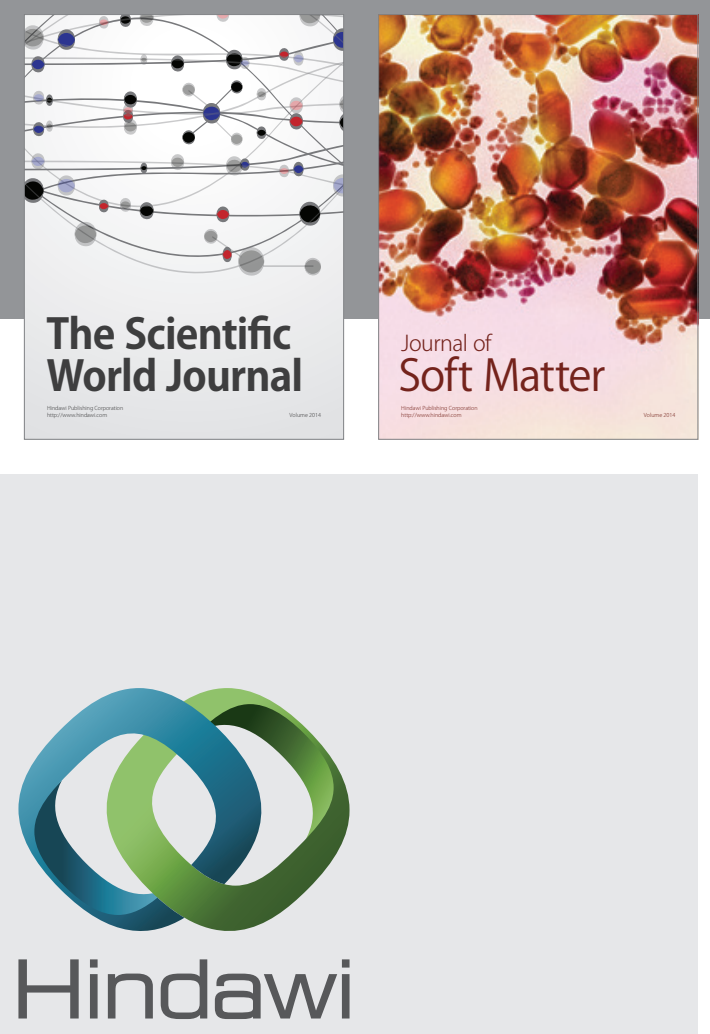

Submit your manuscripts at

http://www.hindawi.com
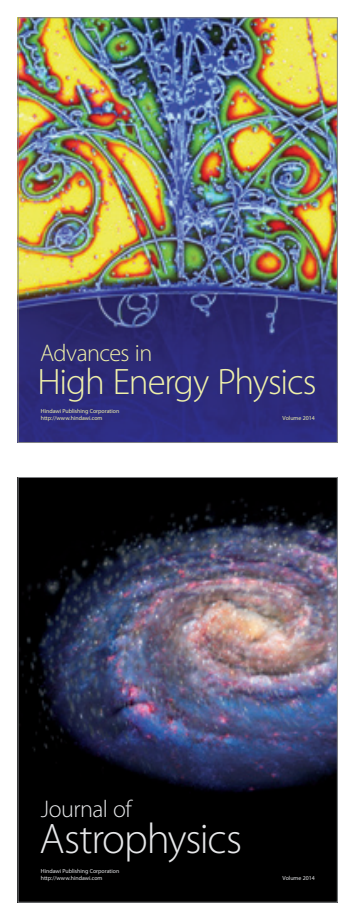
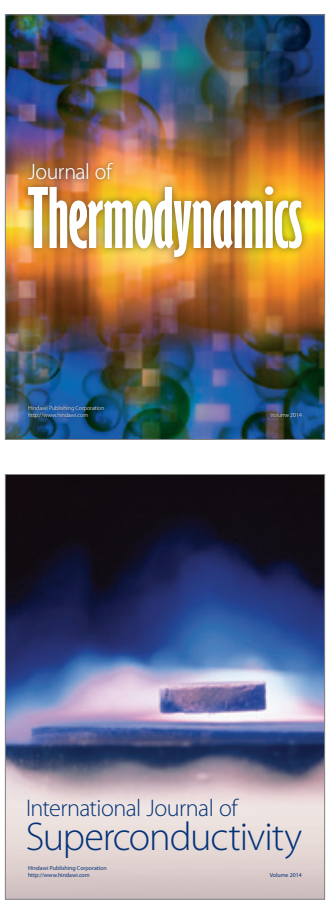
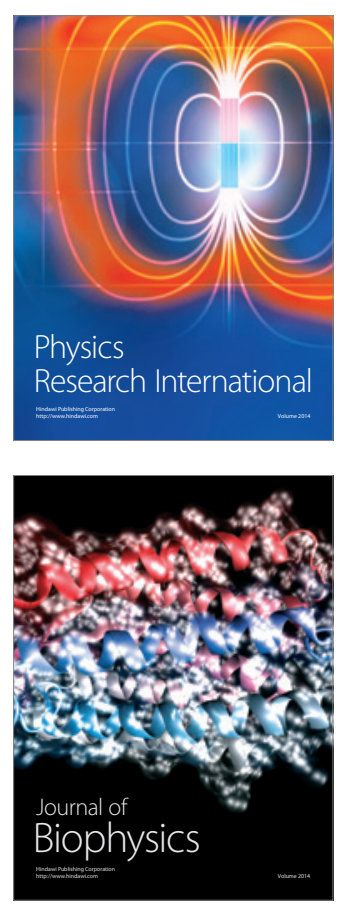
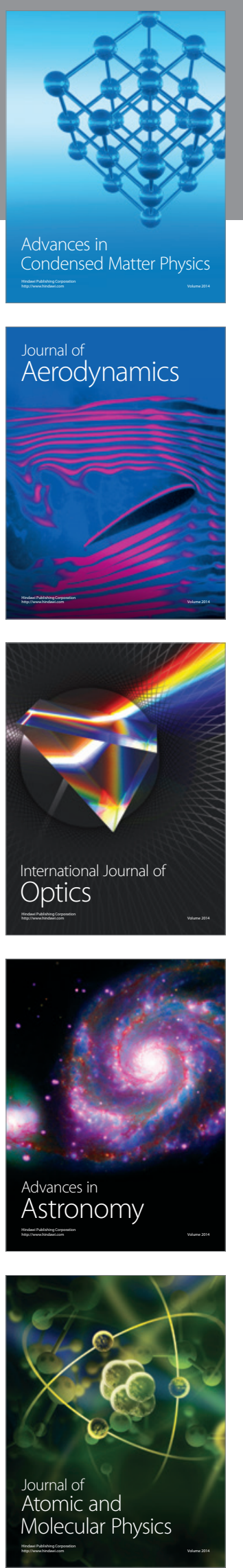